\title{
Impacto de la Reforma Rural Integral sobre la protección de los derechos humanos de la población rural en Colombia*
}

\section{Impact of the Comprehensive Rural Reform on the protection of human rights of the rural population in Colombia}

Diana Alejandra Pulido Buitrago**

\section{Resumen}

El presente artículo realiza una descripción del impacto generado por la Reforma Rural Integral (RRI), establecida por el Acuerdo Final para la Terminación del Conflicto y la Construcción de una Paz Estable y Duradera (AF), sobre la protección de los Derechos Humanos de la población rural en Colombia, cuyo estudio se basa en la transformación de la dinámica social y productiva bajo lineamientos de respeto y promoción de los derechos humanos como elemento imprescindible para conservar el estado de paz planteado por el posconflicto. Por lo anterior, se desarrolla una revisión de la base documental de lo concerniente a la RRI, para, acto seguido, dar cuenta de los derechos humanos, cuya defensa y protección son contemplados dentro de la Reforma, y, finalmente, describir el

Artículo de Revisión

** Profesional en Relaciones Internacionales y Estudios Políticos, Universidad Militar Nueva Granada 
progreso de las acciones establecidas por la RRI sobre la protección efectiva de los derechos humanos.

\section{Palabras clave}

Posconflicto, Acuerdo de Paz, derechos humanos, población vulnerable, ruralidad.

\section{Abstract}

Description of the impact generated by the Integral Rural Reform (RRI) established by the Final Agreement for the Termination of Conflict and the Construction of a Stable and Durable Peace (PA) about the protection of the Human Rights of the rural population in Colombia, whose study it's based on the transformation of social and productive dynamics under guidelines of respect and promotion of Human Rights as an essential element to preserve the state of peace posed by the post conflict. Due to the above, a review of the documentary base regarding the RRI is carried out, for an immediate account of the Human Rights whose defense and protection are contemplated within the Reform and finally, to describe the progress of the actions established by the RRI on the effective protection of Human Rights.

\section{Key words}

Post conflict, Peace agreement, Human Rights, Vulnerable population, Rurality. 


\section{Introducción}

En la actualidad, tras más de medio siglo de conflicto armado, el Estado colombiano se encuentra en un escenario de posconflicto, caracterizado por la expectativa generalizada sobre la efectiva y transparente implementación del Acuerdo Final para la Terminación del Conflicto y la Construcción de una Paz Estable y Duradera (AF), en la medida en que es este el medio que el gobierno y los ciudadanos han considerado acertado para asegurar la paz y el desarrollo de la nación.

Como parte de la ejecución de actividades y compromisos dentro del marco propuesto por la firma del AF entre el Estado y las Fuerzas Armadas Revolucionarias de Colombia - Ejército del Pueblo (Farc-Ep), se establece el curso del Acuerdo sobre la Política de desarrollo Agrario Integral, como herramienta para la transformación del campo según su reactivación a través del desarrollo de la agricultura campesina, familiar y comunitaria, orientada hacia la erradicación de la pobreza rural extrema y la disminución de la pobreza en general en un 50 \% (Oficina del Alto Comisionado para la Paz, 2019).

Es entonces que, la materialización de dicha Política de Desarrollo Agrario Integral se plantea a partir de la Reforma Rural Integral (RRI), la cual deberá

(...) lograr la gran transformación de la realidad rural colombiana para que integre las regiones, erradique la pobreza, promueva la igualdad, asegure el pleno disfrute de los derechos de la ciudadanía y como consecuencia garantice la no repetición del conflicto y la erradicación de la violencia. (Acuerdo Final, 2016, p. 10)

Es por esto que con el objetivo de poder tomar partido en la proyección de acciones a futuro, encaminadas a la consolidación del estado de paz propuesto por el posconflicto, se hace necesario 
examinar su implementación, pues, tal y como lo exponen Ayuso y Parada (2019) "en esta nueva fase es imperativo brindar todas las garantías para la implementación del Acuerdo Final de Paz de forma efectiva, integral y perdurable, si se quiere cumplir con la promesa de una paz sostenible y duradera para los colombianos” (p. 1).

Por lo anterior, es necesario comprender todos los ápices de lo pactado, en referencia a los derechos humanos hacia los que se orienta la RRI y discernir si su ejecución ha sido adecuada y si es capaz de garantizar la no repetición del conflicto; deteniéndose a reflexionar desde el objetivo base del desarrollo rural -mejorar la calidad de vida de los habitantes de las áreas rurales mediante el aumento de sus ingresos según el fortalecimiento de sus capacidades- (Cárdenas Pinzón y Vallejo Zamudio, 2015).

De allí que, a partir de la revisión y análisis de lo contenido en la bibliografía disponible, el presente escrito busca identificar el impacto de la Reforma sobre la protección de los derechos humanos de la población rural en Colombia. Bajo este propósito, se realiza la exposición del escenario en el que se inserta la realidad rural, seguida de la descripción de los principales objetivos y elementos que le componen, para, en seguida, reseñar los DD. HH., cuya protección se contempla en su alcance y, finalmente, analizar los avances materiales conseguidos frente a este respecto años después de la firma del AF.

\section{Ruralidad en Colombia}

Con el objetivo de realizar una aproximación asertiva a la situación de la población rural, es importante partir de la noción de ruralidad, pues su caracterización permitirá alcanzar el objetivo trazado en este documento, reflexionar acerca de la situación de los individuos que la conforman. 
La caracterización de la ruralidad en Colombia surge de la Ley 388 de 1997 a partir de la condición del uso del suelo rural para actividades agrícolas, ganaderas, forestales, de explotación de recursos naturales y actividades análogas. Sin embargo, según lo expuesto en el informe sobre la Misión para la Transformación del Campo del Departamento Nacional de Planeación (DNP), dicha perspectiva ha empezado a considerarse imprecisa en cuanto existen grados de ruralidad según la presencia de dinámicas productivas y el acceso a servicios sociales y de infraestructura; es por esto que para actualizar la clasificación de ruralidad, el DNP ofrece una categorización basada en tres criterios: la ruralidad dentro del Sistema de Ciudades, la densidad poblacional y la relación de población urbano-rural (DNP, 2014).

La ruralidad dentro del Sistema de Ciudades reconoce zonas rurales que pueden aprovechar los beneficios generados por su relación y proximidad con aglomeraciones urbanas; en tanto, la densidad poblacional pretende identificar comunidades rurales con bajos niveles de densidad, y la relación de la población urbano-rural alude a la cantidad de personas que residen en las cabeceras y restos municipales.

La interacción entre dichos criterios arroja como resultado la formulación de cuatro categorías para la caracterización de la población: ciudades y aglomeraciones, intermedios, rural y rural disperso, cuyos atributos se muestran en la Tabla 1 , en relación con la cantidad y distribución de la población. Allí se observa que, para el año 2014, 117 municipios fueron asignados a la categoría de ciudades y aglomeraciones y 314 a la de intermedios, categorías que corresponden a aglomeraciones urbanas con importancia regional y acceso a diversos bienes y servicios. En tanto, 691 municipios con cabeceras pequeñas y áreas no municipalizadas fueron asignadas a la categoría rural y rural disperso; esta última consideración agregada atiende a la reflexión de que la población rural es aquella asentada en la zona 
resto del país ${ }^{1}$, más la contenida en las categorías rural y rural disperso.

Tabla 1.

Categorías de Ruralidad

\begin{tabular}{|c|c|c|c|c|c|}
\hline & & \multicolumn{4}{|c|}{ Población en cabecera (miles) } \\
\hline & & \multirow{2}{*}{$\begin{array}{c}\text { Más de } 100 \\
\text { (Ciudades y } \\
\text { aglomeraciones) }\end{array}$} & \multirow[b]{2}{*}{25 a 100} & \multicolumn{2}{|c|}{ Menos de 25} \\
\hline & & & & $\begin{array}{l}<70 \% \text { población } \\
\text { en zona resto }\end{array}$ & $\begin{array}{l}>70 \% \text { población } \\
\text { en zona resto }\end{array}$ \\
\hline \multirow{4}{*}{ 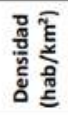 } & $>100$ & \multirow{4}{*}{117} & 29 & 84 & 35 \\
\hline & $50-100$ & & 19 & 132 & 91 \\
\hline & $10-50$ & & 15 & 279 & 203 \\
\hline & 0.10 & & 3 & 54 & 61 \\
\hline
\end{tabular}

Ciudades y aglomeraciones
Intermedios
Rural
Rural disperso
Fuente: DDRS-DNP en el marco de la Misión para la Transformación del Campo

Cabe resaltar que en Colombia la población rural ha disminuido considerablemente, como se muestra en la Ilustración 1 , dado que se encuentra en una etapa de transición demográfica rezagada producto de la disminución de la tasa de fecundidad y la esperanza de vida al nacer; la oferta insuficiente y desarticulada de servicios públicos; el fenómeno migratorio protagonizado por la población activa y el predominio de población potencialmente activa con bajos niveles de escolaridad. Todos estos como factores que contribuyen a perpetuar menores niveles de crecimiento y productividad y, por ende, una mayor incidencia de pobreza.

En Colombia se manejan dos indicadores oficiales y complementarios de pobreza

1 Sobre la distinción hecha entre cabecera municipal -concentración de cualquier número de personas que habitan las ciudades o poblados, donde tenía su sede la autoridad municipal- y resto municipal población que vivía fuera del perímetro de la cabecera municipal- (CEPAL, 2013). 
(...) la pobreza monetaria, que mide el porcentaje de la población con ingresos por debajo del mínimo de ingresos mensuales definidos como necesarios para cubrir sus necesidades básicas y la pobreza multidimensional calculada con el Índice de Pobreza Multidimensional (IPM), que mide los hogares con privaciones en 5 dimensiones básicas de bienestar, distintas a la carencia de ingresos. (DNP, 2017)

\section{Ilustración 1.}

Evolución de la población rural en Colombia

\section{Población rural en Colombia}

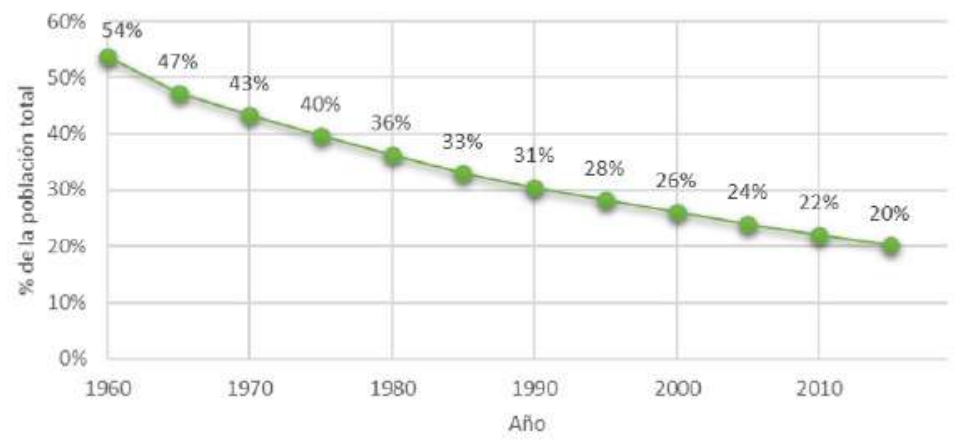

Adaptado de https://datos.bancomundial.org/indicador/SP.RUR.TOTL.

ZS ? locations $=\mathrm{CO}$

Si bien el Banco Mundial establece la proyección de que para el año 2014 la población rural corresponde al 20,2 \% del total de la población en Colombia (Banco Mundial, 2019), considerando la categorización desarrollada por el DNP, la población rural para este periodo asciende al 30,4\%, lo cual permite identificar la significativa porción de población objetivo de la RRI. Lo anterior describe el escenario en el cual se enmarca la Reforma Rural Integral y, por ende, la necesidad final que atiende, la mitigación del fenómeno de pobreza en zonas rurales, esto a través de la consideración del IPM y la perspectiva integral que este envuelve. 


\section{Reforma Rural Integral}

Según el objetivo planteado desde la Política de Desarrollo Agrario Integral, cabe resaltar que el alcance de la RRI estará enmarcado en la ejecución de actividades, tales como el diagnóstico del estado de la población rural, la promoción del acceso a la tierra, la vigilancia de su adecuado uso, la concepción de escenarios de igualdad de oportunidades para individuos rurales, la generación de planes nacionales en respuesta a las necesidades manifiestas y la articulación de agentes de diversa procedencia para la realización de las iniciativas propuestas en lo rural.

Por ende, la RRI deberá garantizar el acceso a la tierra por parte de la población más vulnerable; aprovisionar de bienes y servicios públicos que brinden bienestar y buen vivir a la población rural y asegurar a toda la población colombiana la disponibilidad y acceso suficiente en oportunidad, cantidad, calidad y precio a los alimentos necesarios para una buena nutrición. De allí que su efectividad dependa de la vinculación de las comunidades en espacios participativos y democráticos en los cuales "tengan capacidad de transformación e incidencia en la planeación, implementación y seguimiento de los diferentes planes y programas acordados" (Acuerdo Final, 2016, p. 11).

\section{Principios de la RRI:}

Se establecen como principios de la RRI todos aquellos elementos que sirvan de pilar para el éxito de la misma, como lo son la transformación estructural de escenarios, el desarrollo integral del campo, la regularización de la propiedad, el desarrollo sostenible, la presencia del Estado, la democratización del acceso y uso adecuado de la tierra, así como el impacto y la medición. Igualmente, se cuentan otros aspectos como el bienestar y buen vivir como fines predilectos, la priorización, el restablecimiento, el derecho a la alimentación, la 
articulación y ejecución para los méritos de la igualdad y el enfoque de género, la participación y el beneficio.

\section{Mecanismos de la RRI:}

Son todos aquellos mecanismos que han sido planteados en pro del cumplimiento de los objetivos de la Reforma en cuestión, entre los que se destacan los mecanismos de acceso y uso de la tierra, los Programas de Desarrollo con Enfoque Territorial y los Planes Nacionales para la Reforma Rural Integral, los cuales se reseñan a continuación según lo expuesto en el texto del AF (Acuerdo Final, 2016).

\section{Mecanismos de acceso y uso de la tierra}

\section{Fondo de Tierras para la Reforma Rural Integral}

Fondo creado por el Gobierno Nacional para garantizar el acceso a la tierra por parte de las comunidades rurales más vulnerables, este implica un proceso de distribución gratuito orientado hacia una asignación equitativa de la tierra.

Los terrenos bajo su dominio serán obtenidos a partir de la extinción de dominio de concentraciones ilegales, la recuperación de tierras cuya apropiación resulte indebida tras la actualización catastral, expropiaciones hechas por motivos de interés público, tierras provenientes del robustecimiento de la Reserva Forestal bajo el foco de la sostenibilidad y tierras donadas e inexplotadas según el incumplimiento de su función social y ecológica.

\section{Formalización de la propiedad rural}

Esta medida es contemplada dentro de la RRI con el propósito de prestar protección a los derechos de la pequeña y mediana 
propiedad rural según su propiedad y posesión legitima, pues se espera formalizar cerca de siete millones de hectáreas, prestando especial atención a las zonas en las que se desarrollen PDET y zonas de reserva campesina -que cuentan con especial apoyo por Planes de Desarrollo Sostenible- con miras a mitigar y prevenir prácticas violentas para la protección por mano propia de sus intereses.

Este ejercicio contempla el mantenimiento de la condición de propiedad por parte de campesinos y campesinas, situación que no les excluye de su acceso al Fondo de Tierras en caso tal de contar con un valor menor a una Unidad Agrícola Familiar.

\section{Sistema de catastro e impuesto predial rural}

Es una herramienta de información y planificación promotora del ordenamiento y aprovechamiento productivo del territorio, la restitución y formalización de los derechos de propiedad y el manejo o reducción de la conflictividad por el uso del suelo. Este sistema deberá consolidarse en un plazo máximo de siete años a partir de la actualización del catastro rural ejecutado bajo la autonomía municipal, haciendo especial énfasis en los resultados tempranos que deberá arrojar en las zonas priorizadas.

Lo anterior, bajo un marco de participación colectiva, sumado a la adecuación de la normatividad para que los municipios puedan fijar las tarifas de su impuesto predial en concordancia con "el principio de progresividad: el que más tiene más paga, fundamentado en la equidad y la justicia social” (Londoño Botero, 2017).

\section{Protección de zonas de reserva}

Este mecanismo exige la delimitación de la frontera agrícola y las zonas de reserva para, a su vez, generar alternativas de coexistencia 
entre el ambiente y el bienestar de las personas que habitan o colindan con estas Áreas de Especial Interés Ambiental (AEIA).

Dicha zonificación prevalecerá en zonas priorizadas y servirá de insumo clave para la promoción de áreas rurales y programas de reasentamiento de población. Además, se contemplan otros mecanismos como planes de acompañamiento en vivienda, asistencia y capacitación técnica, promoción del acceso a medios de producción para agregar valor, subsidios y créditos especiales para la compra y leyes futuras en pro de favorecer el acceso a la tierra.

\section{Programas de Desarrollo con Enfoque Territorial}

La RRI prioriza los territorios que han resultado más afectados por el conflicto y espera operar en ellos mediante instrumentos de participación y reconciliación, como son los Programas de Desarrollo con Enfoque Territorial (PDET), los cuales deberán considerar las necesidades y particularidades de cada territorio en el ámbito económico, cultural y social con miras a obtener su sostenibilidad socio ambiental.

Los PDET son un programa subregional de transformación integral del ámbito rural proyectado a 10 años, enfocado en la puesta en marcha de las medidas establecidas por el Acuerdo para los municipios priorizados, bajo el liderazgo de la Agencia de Renovación del Territorio (ART), cuyo origen se remonta a finales de 2015 con el propósito de ayudar a la transformación del sector rural colombiano mediante la disminución de las brechas entre la ciudad y el campo (Alta Consejería Presidencial, 2017).

Considerando el principio de priorización sobre el cual se fundamentan los PDET, caben resaltar las condiciones que caracterizan las zonas prioritarias observadas en la Ilustración 1, como lo son niveles de pobreza y necesidades insatisfechas, afectación derivada 
del conflicto, presencia de economías ilegítimas y debilidad institucional administrativa y de gestión.

\section{Ilustración 2}

Subregiones priorizadas por los PDET

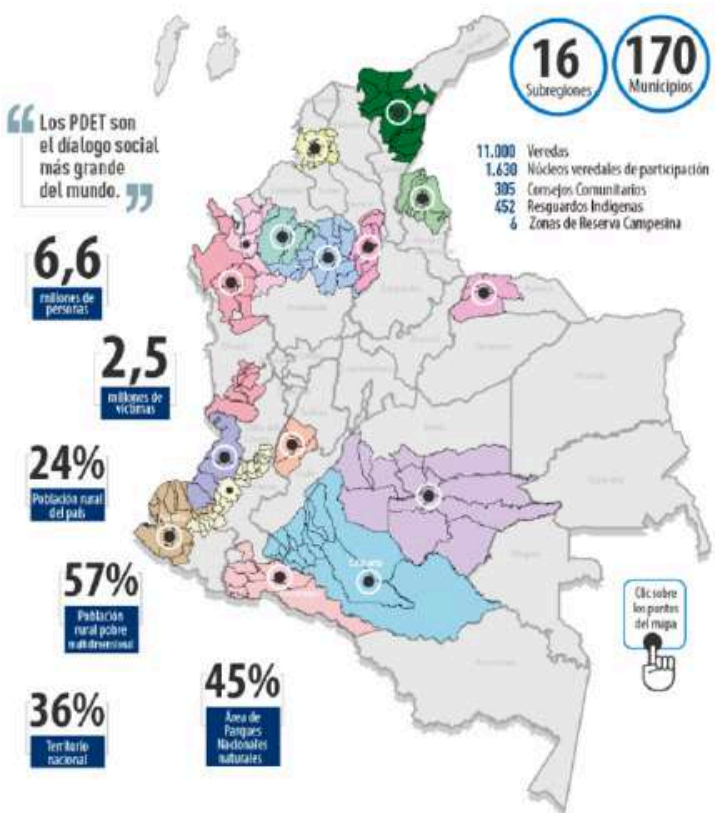

Recuperado de http://www.renovacionterritorio.gov.co/especiales/especial_PDET/ mapa.html

A partir de la articulación propuesta por los PDET, se observan los mecanismos de participación y su sinergia con los niveles territoriales que contempla -Comisión Subregional de Planeación Participativa, Comisión Municipal de Planeación Participativa y la Asamblea Comunitaria, Núcleos Veredales-, situación que permitirá hacer partícipe a la población vulnerable de la formulación de prioridades y la ejecución de herramientas de seguimiento y veeduría de los proyectos. 


\section{Planes de acción para la transformación regional}

Los PDET involucran los Planes de acción para la transformación regional (PATR), espacios participativos que servirán de herramienta clave para el cumplimiento de los objetivos PDET en cada zona priorizada, los cuales estarán compuestos por:

- Enfoque territorial de las comunidades rurales (características socio históricas, culturales, ambientales y productivas de los territorios y sus habitantes, asi como sus necesidades diferenciadas por su pertenencia a grupos en condiciones de vulnerabilidad y la vocación de los suelos) para poder desplegar efectivamente los recursos de inversión pública.

- La ejecución de un diagnóstico objetivo que cuente con la participación de las comunidades para la vinculación de características, necesidades y acciones coordinadas, dirigidas al establecimiento de metas claras y precisas que posibiliten la transformación estructural de las condiciones de vida y de producción.

\section{Proyección de la implementación PDET}

1. Lanzamiento político del PDET.

2. Alistamiento en territorio a través de los niveles de participación (regional, municipal y veredal).

3. Construcción y validación del Pacto Comunitario según el diagnóstico por la Asamblea Comunitaria, alistamiento de la Comisión Municipal y organización de veredas.

4. Construcción y validación del Pacto Municipal de Renovación del Territorio, balance del ejercicio veredal y construcción de insumos. 
5. Construcción y validación del Plan de Acción para la Transformación Regional, mesas de trabajo y consejo regional de planeación.

6. Socialización y apropiación del PATR a nivel regional y nacional.

\section{Planes Nacionales para la Reforma Rural Integral}

Estos planes se erigen como columna vertebral de la RRI, en cuanto se espera que posterior a una fase de transición de quince años se logre erradicar la pobreza, disminuir la desigualdad y mejorar los niveles de vida hacia las metas establecidas.

Lo anterior atiende a la integralidad que se espera abordar desde la composición de dichos planes, condición evidenciada a continuación dada la exposición de los principales elementos de los planes proyectados hacia las áreas de Infraestructura y adecuación de tierras, de desarrollo social, de estímulos a la producción agropecuaria y a la economía solidaria y cooperativa y el sistema para la garantía progresiva del derecho a la alimentación, este último orientado hacia la garantía de la alimentación sana, nutritiva y culturalmente apropiada fomentando el acceso a alimentos de calidad nutricional en cantidad suficiente basado en el incremento gradual de la producción de alimentos y, por ende, de los ingresos; esto requiere compromiso en el cumplimiento de los planes nacionales alineados con políticas alimentarias y nutricionales capaces de monitorear el riesgo y realizar seguimiento a las metas. 


\begin{tabular}{|c|c|c|}
\hline \multirow{3}{*}{ 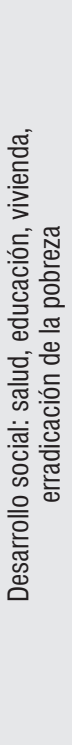 } & 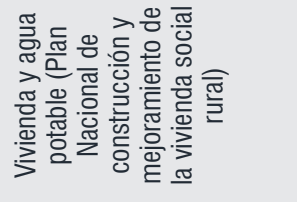 & 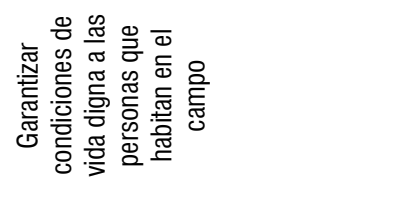 \\
\hline & 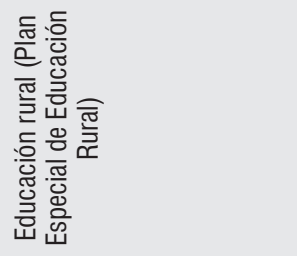 & 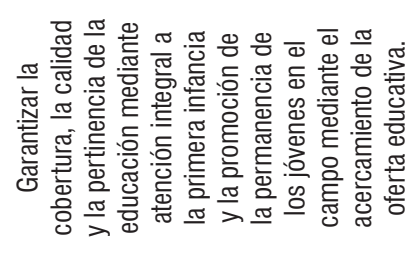 \\
\hline & 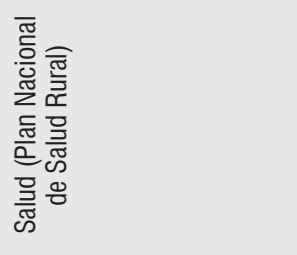 & 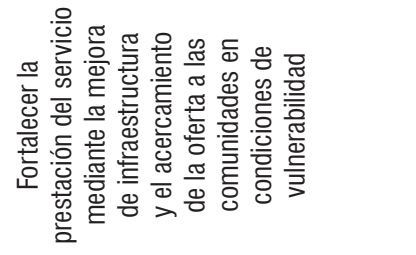 \\
\hline \multirow{4}{*}{ 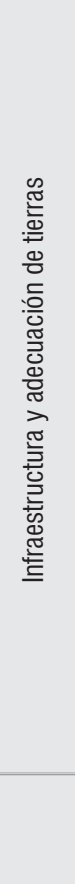 } & 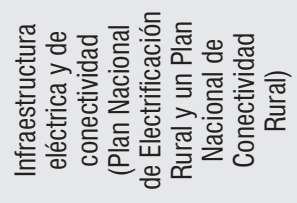 & 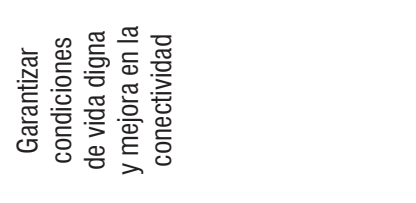 \\
\hline & 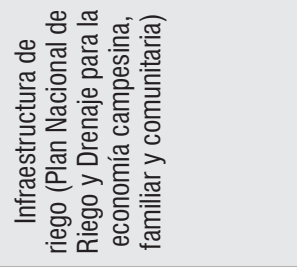 & 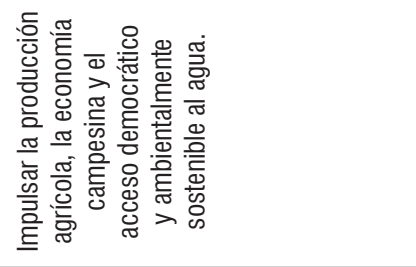 \\
\hline & 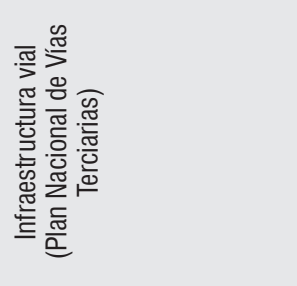 & 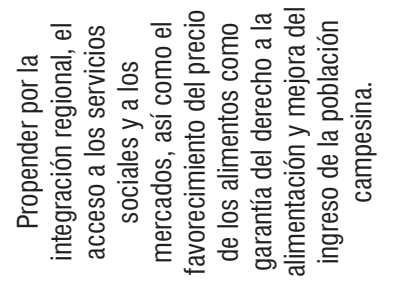 \\
\hline & & $\frac{\stackrel{9}{0}}{\frac{0}{0}}$ \\
\hline
\end{tabular}




\begin{tabular}{|c|c|c|}
\hline \multirow{3}{*}{ 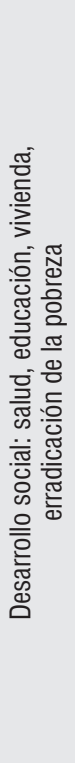 } & 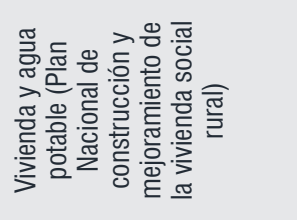 & 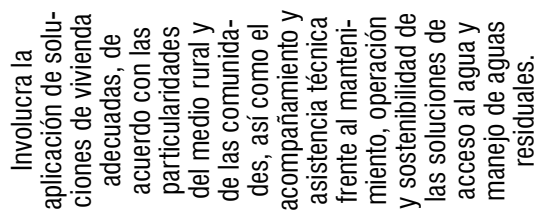 \\
\hline & 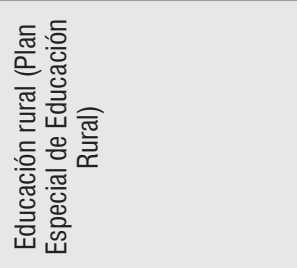 & 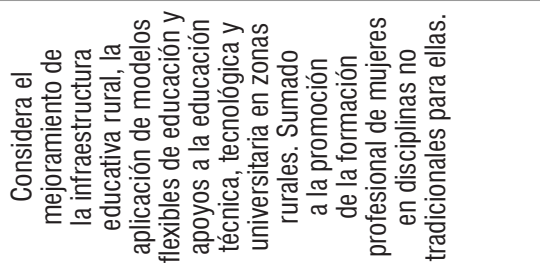 \\
\hline & 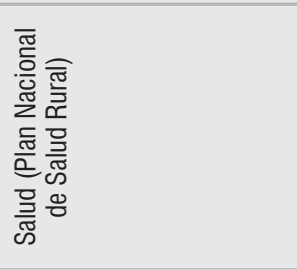 & 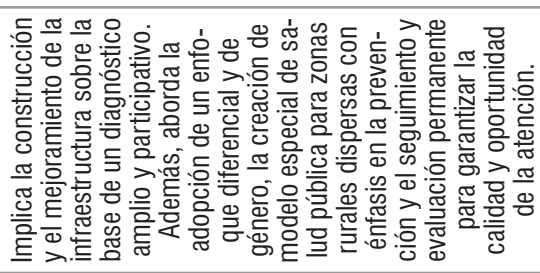 \\
\hline \multirow{4}{*}{ 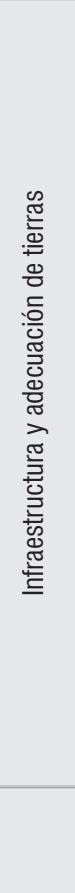 } & 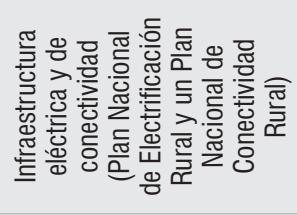 & 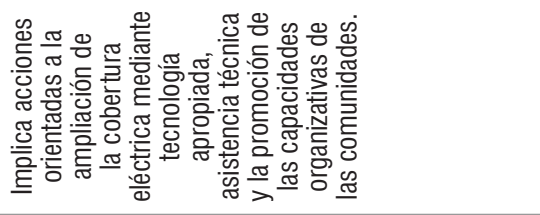 \\
\hline & 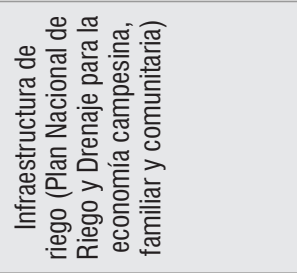 & 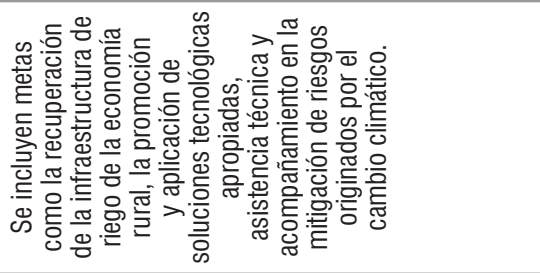 \\
\hline & 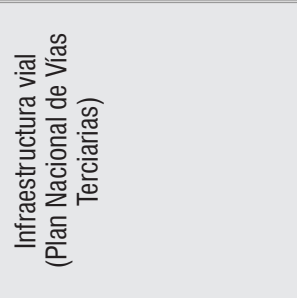 & 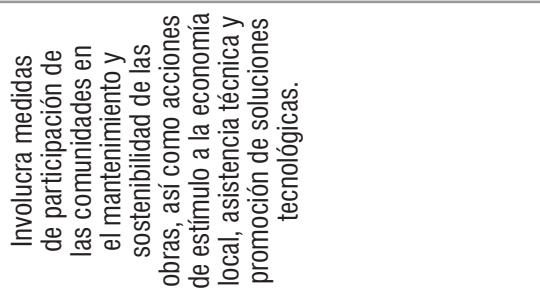 \\
\hline & & 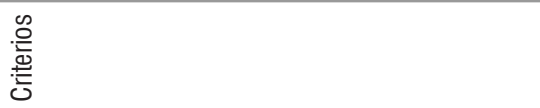 \\
\hline
\end{tabular}




\begin{tabular}{|c|c|c|c|}
\hline \multirow{3}{*}{ 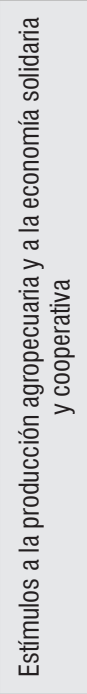 } & \multirow{3}{*}{ 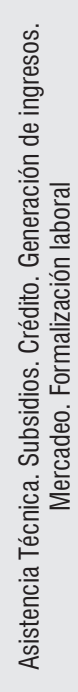 } & 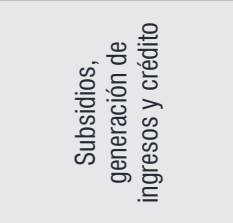 & 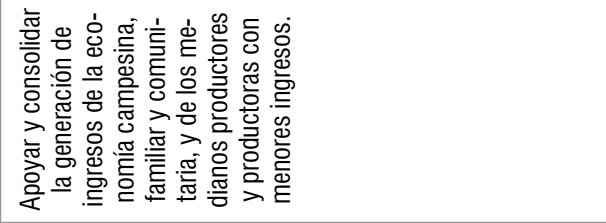 \\
\hline & & 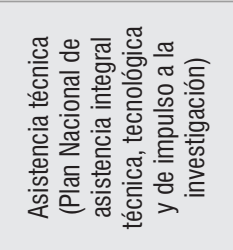 & 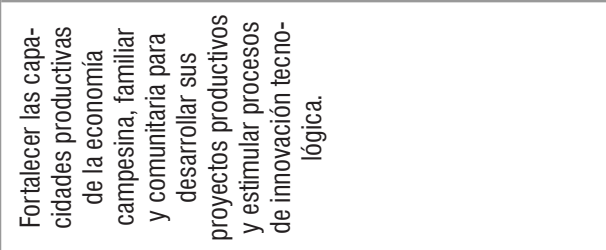 \\
\hline & & 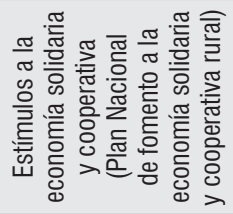 & 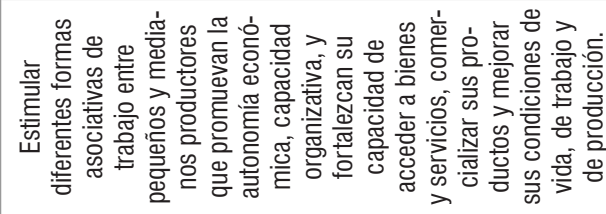 \\
\hline \multirow{3}{*}{ 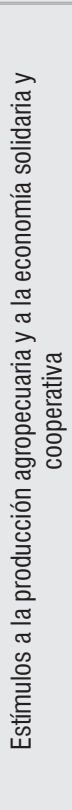 } & \multirow{3}{*}{ 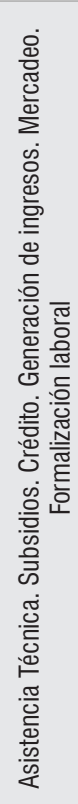 } & 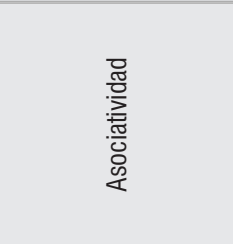 & 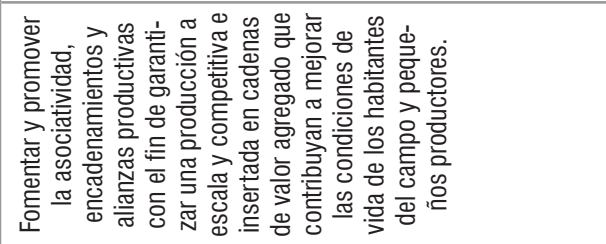 \\
\hline & & 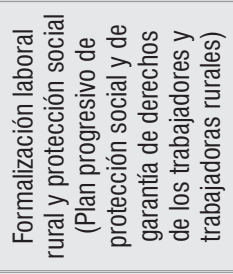 & 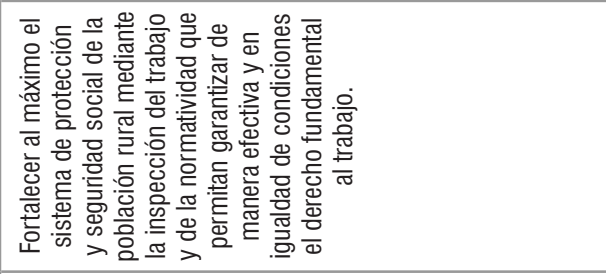 \\
\hline & & 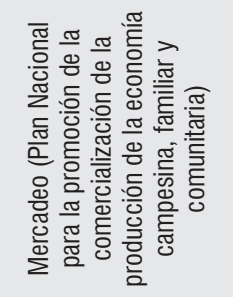 & 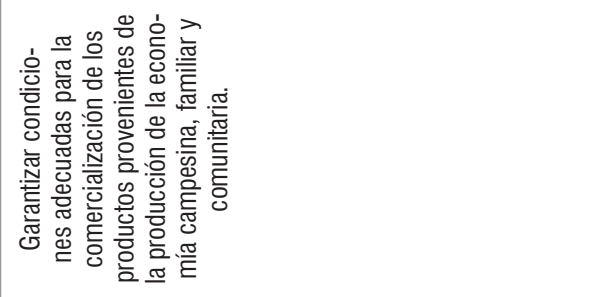 \\
\hline & & & 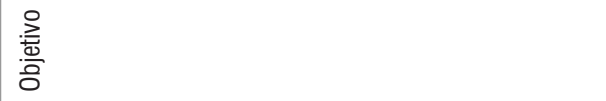 \\
\hline
\end{tabular}




\begin{tabular}{|c|c|c|c|}
\hline \multirow{3}{*}{ 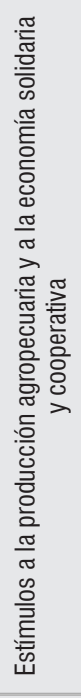 } & \multirow{3}{*}{ 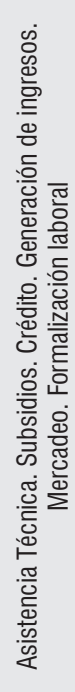 } & 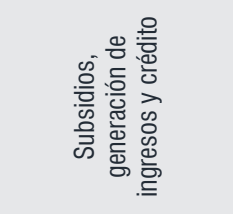 & 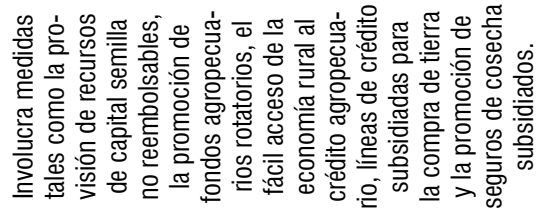 \\
\hline & & 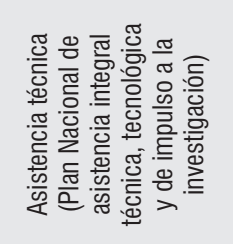 & 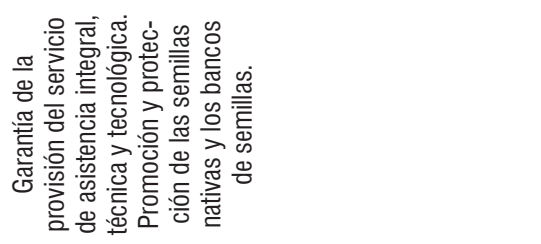 \\
\hline & & 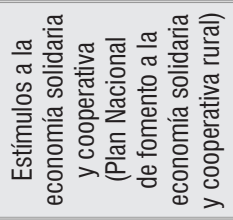 & 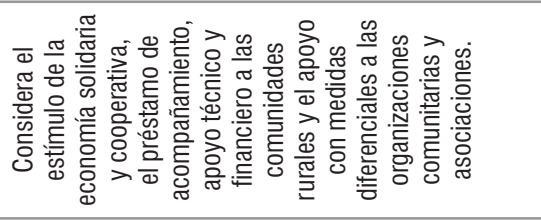 \\
\hline \multirow{3}{*}{ 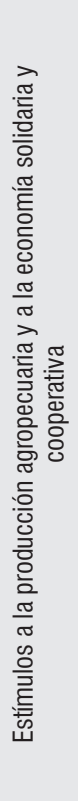 } & \multirow{3}{*}{ 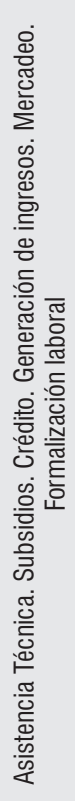 } & 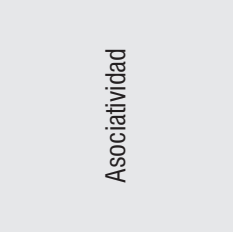 & 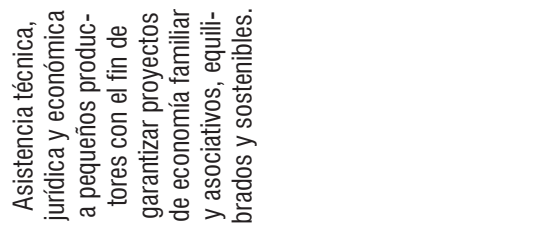 \\
\hline & & 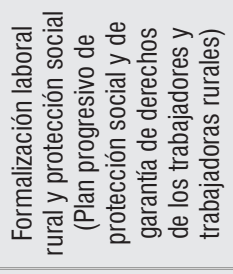 & 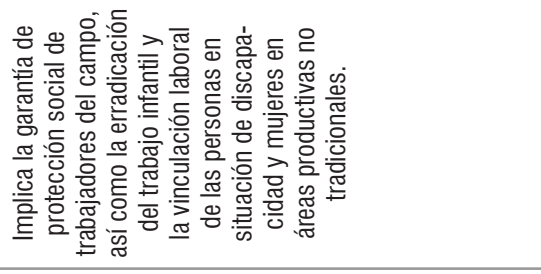 \\
\hline & & 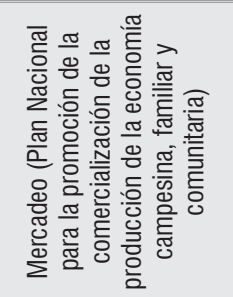 & 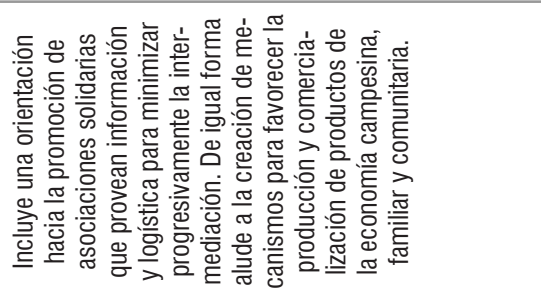 \\
\hline & & & 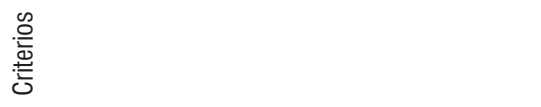 \\
\hline
\end{tabular}




\section{Los derechos humanos en la RRI}

El enfoque de estudio de la RRI desde los derechos humanos se basa en el referente que estos deben ser para dirigir el debate social, ya que las políticas públicas deben orientarse a garantizarlos y evitar su vulneración. Es, entonces, que las políticas públicas como "conjunto de actividades, acciones, planes e instrumentos públicos dirigidos a que el Estado pueda garantizar efectivamente los derechos humanos consagrados en la legislación nacional o en los tratados internacionales" (Consejería DDHH, 2015) deben hacer énfasis en los individuos y en sus condiciones de marginación, exclusión social o discriminación.

De allí que a primera vista se pueda evidenciar el enfoque basado en Derechos Humanos inmerso en la RRI, debido a que sus lineamientos se soportan en el reconocimiento de los individuos como actores clave para su desarrollo, la participación de la población como medio de ejecución y meta final, la concentración de programas en grupos menos favorecidos, así como el análisis basado en todos los actores para determinar las causas inmediatas, subyacentes y básicas de los problemas de desarrollo.

En seguida, para describir brevemente los derechos humanos cuya protección, se encuentra proyectada dentro de lo establecido en la RRI, se presenta la conexión entre estos y los principales criterios de la Reforma.

Propósitos tales como la erradicación de la pobreza rural extrema y la disminución de la pobreza multidimensional en zonas rurales aluden al derecho de los individuos a contar con las mínimas condiciones que les permitan gozar de una vida digna, es decir, se encuentra vinculado al derecho a la igualdad a través del concepto de dignidad; situación que viene siendo destacada como prioritaria desde los objetivos del milenio y que ha sido extendida a los Objetivos 
de Desarrollo Sostenible (ODS), en cuanto establecen como primer objetivo el fin de la pobreza.

Frente a la erradicación del trabajo infantil, cabe resaltar que, como parte de los principios sobre los que se fundamenta el AF, se encuentra la restauración de los derechos de la población vulnerada especialmente de la niñez, de allí que el propender por un escenario en el que la economía del hogar no requiera del trabajo de un menor, se ajusta con el derecho de todos los niños y niñas al goce de una infancia sin abusos.

En tanto, la percepción del campo como un escenario de reconciliación resulta ser un elemento preciso para la no repetición del conflicto y la erradicación de la violencia, pues atiende a la protección del derecho a la seguridad de los individuos que habitan en zonas rurales. Además, el logro y la consumación de dicho escenario se alinea con la necesidad de garantizar a los rurales el derecho a la propiedad individual y al crecimiento económico que le pueda proveer la explotación de la tierra, consideración que es tenida en cuenta en la RRI dentro de la meta de conseguir la asignación y distribución equitativa de la tierra, así como la promoción de un uso adecuado de la misma en cumplimiento de su función social y ecológica; lo cual requiere de un esfuerzo arduo en el cumplimiento de las tareas de formalización y regulación de la propiedad rural, de manera tal que sea posible obtener una efectiva y oportuna aplicación del principio de progresividad.

Según el avance tecnológico que acaece actualmente en el mundo, se hace necesario asegurar el disfrute del desarrollo en ciencia y tecnología por parte de la población, en la medida en que son medios para asegurar el bienestar y la equidad social, de allí que la mejora en la conectividad de las regiones propuesta por la RRI asiste directamente a esta necesidad, dado que se orienta hacia la cobertura adaptada al medio rural, la provisión de asistencia técnica y el acceso 
a internet de alta velocidad en cabeceras municipales, por comunidades vulnerables que por años han permanecido marginadas.

De igual forma, la integración regional que busca la Reforma por medio de un relacionamiento equitativo y la generación de infraestructura vial, con el fin de lograr la convergencia entre la calidad de vida rural y urbana y, a su vez, fortalecer los encadenamientos entre la ciudad y el campo, se direcciona hacia la garantía de la independencia económica y un nivel de calidad de vida justo para la población rural; lo que, sumado al impulso de la producción agrícola a través de la inversión en infraestructura de riego y el acceso sostenible al agua de toda la población, alude al derecho de los individuos a trabajar en condiciones de equidad y en adelante gozar de un medio ambiente sano.

En cuanto al fortalecimiento del servicio de salud, cabe resaltar que la generación y mejora de la infraestructura, la incursión de un modelo especial en salud para zonas rurales dispersas, la incorporación de un enfoque de atención basado en la prevención, la prioridad sobre el mantenimiento de la calidad y la aplicación de un enfoque diferencial sobre cada grupo poblacional se traducen en la protección del derecho de los individuos de contar con los cuidados y asistencia médica especial según la condición que le acaece.

Mientras la garantía de cobertura, calidad y pertinencia de la educación, mediante la generación de infraestructura, la integración de modelos educativos flexibles y la oferta de créditos para el acceso a educación, posee un interés evidente en proteger el derecho de la población rural a la educación, la gratuidad de la instrucción elemental y el acceso equitativo para todos.

Lo anterior favorece, a su vez, la permanencia de los jóvenes en las zonas rurales mediante la aproximación de una oferta educativa integral y de calidad, sumado a la oferta de programas 
e infraestructura orientada al campo de la recreación, cultura y deporte, lo que también permite proteger la riqueza pluriétnica y multicultural y va de la mano con la protección del derecho al bienestar, a elegir su residencia y al libre desarrollo de su personalidad.

Es así que, contar con los bienes (vivienda) y servicios públicos necesarios para garantizar el bienestar de la población rural, así como la disponibilidad y acceso suficiente en oportunidad, cantidad, calidad y precio a los alimentos necesarios para una adecuada nutrición aluden a la provisión de las condiciones necesarias para gozar de una vida digna, siendo además un componente clave de los ODS para la erradicación del hambre.

Es, entonces, que metas como la orientada al desarrollo de la economía campesina y otras formas de economía propia, mediante el estímulo de las formas asociativas de trabajo y su vinculación a proyectos de desarrollo rural, que involucren entre otras cosas el aprovisionamiento de semillas nativas y la asistencia técnica, buscan la independencia económica de los individuos, la agrupación de los individuos en defensa de sus intereses y el mantenimiento de su calidad de vida.

La adopción de un sistema de garantías que facilite el acceso de la economía rural al crédito agropecuario, las líneas de crédito subsidiadas para la compra de tierra, los seguros de cosecha, la garantía de condiciones adecuadas para la comercialización de los productos provenientes de la producción de la economía rural mediante el mercadeo de sus productos y la implementación de sistemas de información para la ejecución de compras públicas y la comunicación de precios regionales son mecanismos enfocados en el favorecimiento de condiciones equitativas y satisfactorias de trabajo. 
Esto, alineado con la mejora del sistema de protección y seguridad social, la incorporación de beneficios económicos periódicos para trabajadores rurales en edad de jubilarse, la protección de la mujer trabajadora y la promoción de la vinculación laboral de personas en condiciones de discapacidad y de mujeres en áreas no tradicionales son metas que velan por la protección del derecho a la igualdad y a la protección de los individuos en condición vulnerable.

A lo largo de lo decretado en el primer punto del AF se establecen compromisos explícitos con respecto a problemáticas ambientales, lo que, tal y como lo exponen Gallego, Azcarate y Kornov (2019), demuestra un reconocimiento temprano por parte del gobierno y las FARC de la prioridad sobre la protección del medio ambiente, debido a que el desarrollo en las zonas posteriores al conflicto debe realizarse de manera sostenible (Gallego Dávila, Azcárate y Kornov, 2019), de allí que, además de dirigirse hacia la protección del derecho a gozar de un medio ambiente sano, este avance se dirige hacia la construcción de los medios necesarios para ello de modo participativo, a través de elementos como los PDET y en ellos los PATR.

\section{Implementación de la RRI en Colombia}

La revisión del estado de la implementación de la RRI es crucial para conocer el impacto material de esta sobre la protección de los derechos humanos de la población rural en Colombia; es por esto que su aplicación es foco de numerosas opiniones acerca de la complejidad que entraña y la cuestionada velocidad de su ejecución.

Para proceder al examen de la implementación de la Reforma, inicialmente, se toma en consideración el último reporte ofrecido por el gobierno firmante del AF en mayo de 2018, el informe de Rendición de Cuentas "Construcción de Paz" elaborado por el Departamento Administrativo de la Presidencia de la República (DA- 
PRE, 2018), en el que se notificaron las acciones que aportaron a la construcción de paz en Colombia desde el marco de la RRI.

Tal reporte alude únicamente al fortalecimiento territorial para la implementación de la Política de Estado para el Desarrollo Integral de la Primera Infancia "De Cero a Siempre", orientada hacia la garantía de los Servicios de atención integral a la primera infancia en zona rural. Dicho avance contempló ejercicios valiosos de construcción y acompañamiento en territorio, los cuales arrojaron como retos el lograr mayor estabilidad de los equipos locales encargados de la implementación, hacer más fuerte la articulación departamento- municipio y avanzar en una gestión diferencial según las características de los niños y niñas del territorio.

El desarrollo descrito estuvo apoyado en que una vez cumplidos los plazos de implementación se lograse atender integralmente al $100 \%$ de los niños y niñas menores de 6 años que habitan las zonas rurales, es decir, que a 2031 cada niño contase con "registro civil de nacimiento, afiliación al sistema de salud, esquema de vacunación completo, consultas de crecimiento y desarrollo, valoración y seguimiento nutricional, acceso a educación inicial de calidad, acceso a colecciones de libros y contenidos especializados para la primera infancia” (DAPRE, 2018, p. 12).

A principios del año 2019 el Instituto Kroc emitió la actualización de su Informe "Hacia una paz de calidad en Colombia", en el cual expuso que más de dos tercios de los compromisos pactados en el AF se encuentran en progreso o ya fueron completados, además argumenta que el grado de avance ha sido significativo, incluso considerando las demoras esperadas por el cambio de gobierno, desde la posesión del actual presidente Iván Duque.

Sin embargo, el mismo informe comunica que de lo concerniente al primer punto del AF solo el $3 \%$ de lo pactado se ha ejecutado 
por completo, en tanto un $8 \%$ se encuentra en estado intermedio y un $51 \%$ ha sido implementado mínimamente, mientras resta por iniciar la implementación del 38\% (Instituto Kroc, 2019). De allí que, si bien el Fondo de Tierras, los PDET y los Planes Nacionales han sido abordados, su materialización cuenta con significativos hallazgos que evidencian la necesidad de mayor laboriosidad en su gestión, pues a pesar de la positiva percepción obtenida por la implementación general del AF, lo relacionado con la RRI presenta considerables demoras.

En seguida, se describe el avance de la materialización del Acuerdo con base en elementos claves expuestos en el informe emitido por la Procuraduría General de la Nación en el primer semestre de 2019 (Procuraduría General de la Nación, 2019) y en el Sexto informe de verificación de la implementación frente a la ejecución del AF (CINEP - CERAC, 2019), los cuales permitirán profundizar en el análisis sobre la implementación de la RRI.

Es así que se destaca la aprobación de la Línea Especial de Crédito en el marco de la RRI para la compra de tierra de uso agropecuario y hasta la cobertura de costos de formalización para mujeres rurales y pequeños productores a través de la Comisión Nacional de Crédito Agropecuario. A pesar del estado incipiente de esta medida, es necesario considerar de antemano la meticulosidad con la que se debe aplicar, según se garantice sea una medida bien direccionada, sostenible y efectiva, pues, como comentan Boron, Payán, MacMillan y Tzanopoulos (2016), existe el riesgo de beneficiar a unas cuantas actividades relacionadas con usos específicos de la tierra, cuestión que para la línea especial de crédito terminaría por sesgar su enfoque y disminuir su impacto socioeconómico, llegando incluso a afectar el equilibrio de ecosistemas, su biodiversidad y sus recursos.

Para la comprensión de lo ya ejecutado del AF, es necesario considerar el Sistema Integrado de Información para el Posconflicto 
(SIIPO), ordenado en el año 2018 para hacer seguimiento a la implementación de las acciones derivadas del AF, frente al cual cabe resaltar que en lo concerniente a la línea de crédito especial no se encuentra información.

Sumado a esto, se enuncia un deficiente desarrollo de los mecanismos de conciliación y resolución de conflictos de uso y tenencia, de prevención e impulso de solución a los conflictos entre vocación y usos del suelo y de concertación y diálogo social, resaltando que no hay ningún avance reportado ni se cuenta con registro en el SIIPO.

En tanto, la erradicación de la pobreza extrema en el ámbito rural presenta resultados satisfactorios, en cuanto la meta de llegar al nivel de 4,0 \% para el año 2031, según lo contemplado en los ODS, a partir de la incidencia del $18,1 \%$ de la pobreza monetaria extrema rural registrada en 2016, implicaría una reducción promedio anual de 0,9 puntos porcentuales, lo que se encuentra por debajo del promedio de la reducción anual actual de 1,4 puntos porcentuales. Situación que se ve opacada por los resultados del objetivo de reducir en un $50 \%$ el índice de pobreza multidimensional (IPM), debido a que según el IPM de 2016 de 37,6 \% se tiene que para 2031 este debería ubicarse en $18,8 \%$ lo que aduce a una reducción anual de $1,25 \%$, cifra bastante superior al promedio anual actual de $0,9 \%$.

De cara al fondo de tierras, se tiene que, al corte de marzo de 2019, 1753 hectáreas fueron entregadas a través del Fondo, las cuales fueron tramitadas mediante 253 resoluciones de adjudicación, lo que se traduce en apenas el 0,058 \% de la meta del Plan Marco de Implementación $^{2}$ (PMI) de administrar 3 millones de hectáreas, lo

2 El PMI, según el AF y el CONPES 3932, es la guía para la implementación en un horizonte de 15 años y es la base para la articulación con los Planes Nacionales de Desarrollo, Planes de Desarrollo Territorial y herramientas de programación presupuestal y seguimiento. 
que, a su vez, corresponde al $0,32 \%$ de las hectáreas que ingresaron al fondo a 31 de marzo de $2019^{3}$ (DNP, 2016).

Es así que la gestión del fondo de tierras se cataloga como precaria, pues la caracterización e ingreso de tierras resulta ser un proceso lento y engorroso, ya que no se tiene certeza de la condición adjudicable ${ }^{4}$ de los predios; verbigracia se presentan predios en Bolívar y Córdoba, ubicados en Islas del Rosario y San Bernardo, los cuales ya contaban con la condición de inadjudicable previo a su ingreso al fondo, además de otros tantos casos en los que la dilación sobre el proceso de caracterización ha dificultado conocer su situación registral, catastral y la presencia de asentamientos y Unidades Agrícolas Familiares. Si bien se había planteado la construcción de una estrategia para conseguir la máxima contribución de cada una de las fuentes proyectadas del Fondo de tierras, aún no se evidencian muestras de ello.

Igualmente, ante la formalización de hectáreas de pequeña y mediana propiedad al mismo corte de marzo de 2019, se registra la formalización de 5831 predios correspondientes a 7374,73 hectáreas, lo cual equivale, a su vez, al 0,105 \% de la meta de 7 millones de hectáreas trazada con anticipación en el AF. En esta como en otras medidas la información de formalización no se encuentra en el SIIPO.

3 Según la Procuraduría General de la nación, del total del área ingresada al Fondo de Tierras el 1,2 \% corresponde a bienes de extinción judicial de dominio transferidos por la Sociedad de Activos Especiales (SAE), 1,1 \% a procesos agrarios de extinción administrativa de dominio por incumplimiento de la función social y ecológica, 39,8 \% a predios del inventario de bienes del antiguo Fondo Nacional Agrario y el 57,9\% a baldíos. Los departamentos con más tierras ingresadas al fondo son Bolívar (46,97 \%) y Meta $(9,47 \%)$.

4 Los baldíos inadjudicables corresponden a parques naturales nacionales, predios seleccionados para planes viales, donde se adelanten explotaciones de recursos naturales, sabanas y playones comunales que periódicamente se inunden, y áreas de reserva forestal de la Ley $2^{\mathrm{a}}$ de 1959. 
Es necesario resaltar que el proceso de entrega de tierras cuenta con un procedimiento administrativo de registro de Sujetos de Ordenamiento (RESO) ${ }^{5}$, el cual supone un gran progreso en el control y monitoreo de los beneficiarios y otorga transparencia al proceso, esto a pesar de que el mismo ha registrado 45666 solicitudes de acceso a tierra y 7407 de formalización de la propiedad rural, frente a los cuales solo el 7,4 \% de las solicitudes de acceso han sido tramitadas.

En temas de gestión catastral según el Ministerio de Ambiente a julio de 2018, se habían zonificado 139 municipios, mientras que previo a ello se esperaba contar con 202, entre ellos todos los 170 municipios PDET. Si bien se ha realizado la articulación institucional necesaria para establecer y ejecutar el sistema multipropósito para el año 2023, aún hoy no ha sido posible llegar a la meta del piloto proyectado para el año 2017 sobre 23 municipios; cabe resaltar que dicho piloto es imprescindible en la medida en que sus resultados permitirán estimar acertadamente costos, tiempos y condiciones técnicas a utilizar en la aplicación de la estrategia para la implementación de la política pública de Catastro Multipropósito, avalada por el CONPES 3958 DE 2019 que propone un plan de acción a siete años (2019-2025).

En cuanto a la protección de zonas de reserva, se observa un avance considerable, ya que se encuentran actualizadas las AEIA bajo una nueva clasificación y tipología, permitiendo contar con una metodología para identificar la frontera agrícola y su diferenciación de zonas de reserva y ecosistemas estratégicos ${ }^{6}$; lo anterior resulta muy favorable, dado que las actividades ya realizadas se constituyen como elementos base para la planificación del uso de la tierra, práctica

5 Sistema encargado del registro de sujetos beneficiarios potenciales del fondo y la identificación de requisitos de las familias campesinas y comunidades étnicas que aspiran a programas de acceso y formalización de tierras.

6 Los ecosistemas estratégicos abarcan paramos, humedales, bosques secos y otros bosques altos densos. 
implícita en la RRI que a su vez favorece la seguridad alimentaria y la equidad social (Boron, Payán, MacMillan y Tzanopoulos, 2016).

En relación con los seguros de cosecha, sólo se cuenta con el escaso avance en la elaboración de un documento de seguro catastrófico que cubre a la población que se enmarca dentro de la Agricultura Campesina Familiar y Comunitaria.

Frente a los PDET la Procuraduría reafirma el gran avance que estos significan, sobre cuyo ejercicio se resalta la construcción de los pactos comunitarios y municipales los cuales permitieron aproximarse al territorio y favorecieron la formulación de los PATR; lo anterior, además de arrojar la compilación de iniciativas para la transformación del territorio provenientes de él mismo, permitió visibilizar el quehacer institucional del Gobierno y de esta forma a las comunidades vislumbrar la posibilidad de demandar la garantía de sus derechos.

Los PATR presentan un total de 32.808 iniciativas las cuales se agrupan en ocho pilares, cuyo análisis ha permitido identificar una alta coincidencia entre estas iniciativas y la gestión de la Metodología General Ajustada (MGA) la cual corresponde a los productos concebidos por las entidades públicas en sus proyectos de inversión. En la Ilustración 2. Iniciativas Planes de Acción para la Transformación Regional se observa la distribución de iniciativas entre los pilares.

Lamentablemente este proceso de diseño de los PATR, resultó estar desprovisto de límites básicos frente al uso del recurso presupuestal, de coherencia con la oferta disponible y por ende de condiciones mínimas de viabilidad; es así que la Procuraduría establece que una considerable proporción de los PATR carecen de orden y esquema al no contar con recursos, cronogramas, responsables y metas coherentes. 


\section{Ilustración 3.}

Iniciativas Planes de Acción para la Transformación Regional

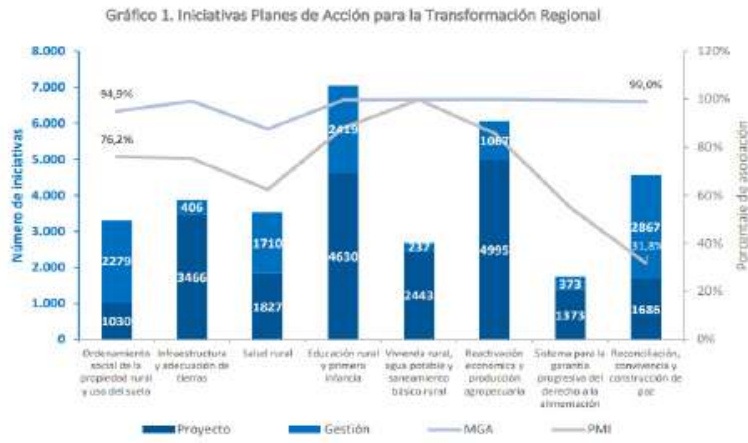

Fuente: Recuperado de Primer Informe al Congreso sobre el estado de Avance de la Implementación del Acuerdo de Paz 2016-2019.

Las obras PDET que cuentan con 3 fases de ejecución y que fueron concebidas como el modo de atender en el corto plazo las principales necesidades de comunidades rurales cercanas a los Espacios Territoriales de Capacitación y Reincorporación (ETCR) y las zonas afectadas por cultivos de uso ilícito, actualmente cuentan con 1.237 proyectos en fase 1 y 2 (622 terminados, 185 en ejecución, 328 por iniciar ejecución y 102 en reestructuración), en tanto la fase 3 cuenta con 2.799 iniciativas cuya ejecución aún no ha iniciado.

Conforme los Planes Nacionales se conciben mediante su acción conjunta en pro de conseguir en un periodo de 15 años la erradicación de la pobreza extrema y la reducción del IPM rural en un 50\%. Cabe resaltar que la meta de contar dichos planes adoptados para el año 2018, aun no se cumple a cabalidad, puesto que ya en el año 2019, 14 de estos aún no habían sido expedidos cuando debieron haber sido adoptados previo a la construcción de los PATR; lo que resulta crítico ya que la necesidad de contar con los Planes Nacionales es poder dar alcance a la RRI hacia toda la ruralidad y no solo hacia las áreas priorizadas. 
Es, entonces, que solo dos Planes Nacionales han sido adoptados, mientras varios aún no concluyen su diseño y, por ende, no se cuenta con su implementación. En seguida se reseñan algunos avances de los ya aprobados, como el Plan Nacional de Vías para la Integración Regional (PNVIR), el cual involucra 131 corredores priorizados en 170 municipios, es así que ya el $100 \%$ de las vías priorizadas se encuentran construidas o en mantenimiento; así como el Plan Nacional de Electrificación Rural (PNER), por el que a 31 de marzo de 2019 se brindó servicio de energía eléctrica a 12764 nuevos usuarios (54,01 \% en municipios PDET), lo cual se encuentra por debajo de las metas proyectadas.

Además, frente a la capacidad instalada de fuentes no convencionales de energía y de soluciones de tipo híbrido en las Zonas No Interconectadas (ZNI) con corte a 31 de marzo de 2019, el PNER llegó a contar con una capacidad instalada de 4,51 megavatios a nivel nacional y 3,00 megavatios en municipios PDET, valores que también se encuentran por debajo de las metas para el año 2018 (10,59 a nivel nacional y 6,66 en los PDET). En relación al objetivo de personas capacitadas a través de asistencia técnica, para el mantenimiento y sostenibilidad de las obras se tiene una meta para el año 2022 de 19776 personas, frente a lo que cabe resaltar que en el primer trimestre de 2019 se registraron solo 1197 personas capacitadas.

Finalmente, la evaluación hecha por la Procuraduría sobre los indicadores del PMI, en los que se soporta la intervención planteada desde la RRI, permite deducir una falta de avance frente a los términos y criterios de ejecución del primer punto del AF, en cuanto solo 8 de los 24 indicadores asociados cuentan con ficha técnica aprobada y reposan en el SIIPO. Además, como se verá más adelante solo 9 de estos cuentan con un equivalente en el PND y 10 de los 15 cuya medición acabó en 2018 no han sido cumplidos. 
Lo anterior, aunado a que aún hay temas como la legislación sobre jurisdicción agraria, la cual tiene un grado de avance nulo, muy a pesar de lo pactado previamente en el AF de aprobar en los primeros doce meses la legislación necesaria para la implementación del Acuerdo; elemento frente al cual cabe considerar la posición de Locatelli, Aldunce, Fallot y Le Coq (2017), quienes comentan que este tipo de medidas legislativas requieren del apoyo oportuno y permanente del sector académico, esto a través de canales de comunicación efectivos para la provisión de un mayor respaldo al desarrollo de política desde la perspectiva del concepto y la teoría, manifestando nuevamente la necesidad de un abordaje integral de lo pactado en el AF (Locatelli, Aldunce, Fallot y Le Coq, 2017).

\section{Incorporación de la RRI en el Plan Nacional de Desarrollo 2018-2022}

La implementación a futuro de la RRI depende de la articulación de lo contenido en el AF dentro del Plan Nacional de Desarrollo (PND), pues este se constituye en el marco de acción a nivel nacional. De allí que en seguida se examine la dinámica establecida por el gobierno actual para el cumplimiento de lo acordado, a la luz de lo trazado por el gobierno en curso y lo expuesto en el informe de Verificación de la implementación del Acuerdo Final de Paz en Colombia (CINEP - CERAC, 2019).

Partiendo de lo dispuesto en el Acto Legislativo 01 de 2016, en el cual se ordena incluir un componente específico para la paz en el Plan Plurianual de Inversiones del PND como forma de garantizar los recursos para la implementación del AF, se evidencia un cumplimiento formal y solo parcial, ya que en el actual PND se encuentra incluido un aparte llamado Plan Plurianual de Inversiones para la Paz, cuya proyección no corresponde a un plan cuatrienal que posibilite la implementación de lo pactado. 
El PND establece como pilares la legalidad y el emprendimiento para el alcance de la equidad a partir de los Pactos que esquematiza, como lo son el Pacto por la Legalidad, orientado a garantizar la convivencia que permita a los colombianos vivir con libertad y en democracia; el Pacto por el Emprendimiento y la Productividad de las regiones, el cual propende por una transformación productiva que derive en una economía dinámica, incluyente y sostenible y el Pacto por la Equidad que busca la igualdad de oportunidades. Sumado a esto y como herramientas habilitantes, el PND contempla los pactos por la descentralización, la transformación digital de Colombia, los recursos minero-energéticos para el crecimiento sostenible y la expansión, el pacto por la sostenibilidad, la ciencia, la tecnología y la innovación; además de otros pactos, como el de transporte y logística para la competitividad y la integración regional, de la calidad y eficiencia de los servicios públicos, de la protección y promoción de nuestra cultura y desarrollo de la economía naranja, de la construcción de paz, de la equidad de oportunidades para grupos étnicos, de la inclusión de todas las personas con discapacidad, de la equidad de las mujeres y por una gestión pública efectiva (PND, 2018).

En referencia a la vinculación de lo establecido en el AF dentro del PND, se observa que, dentro del pacto por el emprendimiento, la formalización y la productividad se estructuran acciones que apuntan hacia una meta de títulos formalizados que otorguen el acceso a tierras y sobre predios privados; asimismo, el pacto por la equidad de oportunidades para grupos étnicos se enfoca en el otorgamiento de títulos colectivos y programas de acceso a la tierra.

Frente a la educación de calidad, el pacto por la equidad aún no instaura una guía de acción, pues afirma que el Ministerio de Educación Nacional trabajará en la definición de una línea base y el establecimiento de una meta de alfabetización en lo rural; de allí que a pesar de sugerir un incremento a la meta pactada por el $\mathrm{AF}$, en cuanto al nivel de educación inicial, estos dos lineamientos 
dados al Ministerio generen alta incertidumbre al no contar previo a su establecimiento con una meta puntual ni una tasa de cobertura adecuada para su seguimiento.

Se destaca que, dentro del Pacto por la sostenibilidad, se encuentran acciones orientadas hacia el incremento del $20 \%$ sobre la efectividad del manejo de áreas protegidas públicas y áreas con esquemas de pagos por servicios ambientales e incentivos a la conservación; en tanto, el Pacto por la construcción de paz posee un enfoque en alcanzar un $43 \%$ de las iniciativas PDET implementadas (viabilidad garantizada), un IPM de $28,2 \%$ sobre la línea base de $40,4 \%$ y el $40 \%$ de hogares rurales con acceso a agua potable en zonas PDET.

Además, cabe resaltar que el enfoque étnico propuesto por el AF se encuentra incluido transversalmente, tal y como se observa en el caso del Pacto por la equidad y el Pacto por la construcción de paz; incluyen estrategias transversales relacionadas con el mejoramiento de las condiciones de vida y el mejoramiento de la visibilidad estadística de los grupos, su territorialidad colectiva, su producción y conservación, su conectividad territorial, el grado de apoyo al desarrollo cultural, el estado de participación, convivencia y construcción de paz de la población, mediante el enfoque sobre comunidades indígenas, Rrom y negras, afrocolombianas, raizales y palenqueras.

De igual forma se observa cómo el PND aborda algunos aspectos de la RRI, como el Sistema Catastral Nacional Multipropósito y los mecanismos de formalización de la tierra; sin embargo, dicha integración no es del todo satisfactoria, en cuanto la meta de completar el catastro a nivel nacional para 2023, se reduce a lograr el $60 \%$ de la actualización catastral para el año 2022 y alcanzar un sistema de catastro multipropósito completamente implementado en 2025 bajo el marco del CONPES 3958. 
De igual forma, el PND se queda corto frente al acceso a tierras y el Fondo de tierras, puesto que las metas que establece se encuentran en términos de número de predios y solicitudes, en tanto el AF plantea sus metas en número de hectáreas; a lo que se suma la reducción de la meta frente a la formalización de tierras, debido a que el AF plantea 7 millones de hectáreas y el PND prevé tan solo 9800 hectáreas de propiedad privada rural formalizadas (Secretaría Técnica del Componente Internacional de Verificación CINEP/ PPP-CERAC, 2019).

Además, frente a la incorporación del ítem de la disminución y erradicación de la pobreza, en el PND se encuentra que mientras el AF establece la erradicación de la pobreza extrema y la disminución en $50 \%$ de la pobreza multidimensional ambas en zonas rurales, el PND aborda la disminución de la pobreza extrema en términos generales sin hacer énfasis en lo rural y mucho menos en las zonas priorizadas. De igual forma, se encuentran discrepancias en la premisa de la RRI de erradicar el analfabetismo para 2031, dado que el PND no toma partido en el establecimiento y proyección de una meta, en cuanto asigna al Ministerio de Educación la definición de un objetivo a partir de los resultados del Censo Nacional de Población de 2018.

Tal y como se comentaba previamente, en lo concerniente a los PDET actualmente se cuenta con los 16 PATR elaborados y aprobados, cuestión de gran relevancia dado el papel clave de los PDET como articulador de la RRI, de allí que sea prudente resaltar el ejercicio propuesto por el PND para maniobrar la materialización de estos planes mediante la incorporación de la Hoja de Ruta Única (HRU) como apuesta para la implementación efectiva de los PDET en los 170 municipios priorizados, junto con los Planes Nacionales Sectoriales (PNS), los Planes Integrales de Reparación Colectiva 
(PIRC), Plan Marco de Implementación (PMI), Planes territoriales y Planes de las Zonas Estratégicas de Intervención Integral (ZEII $)^{7}$.

Es por esto que sobresale el valor de la propuesta de la HRU al constituirse como un mecanismo valioso para la protección de los derechos humanos de la población rural, en la medida en que su objetivo es lograr armonizar los diferentes instrumentos aplicables al direccionamiento del campo, en pro de concretar sus esfuerzos, orientar sus recursos y conseguir un beneficio integral de la población a la cual se encuentra dirigido. Lamentablemente, dicha articulación pierde valor, pues si bien el PND establece metas de reducción de pobreza y otras relacionadas con la calidad de vida en los municipios PDET, no es posible identificar el enfoque sobre los municipios PDET en todos los pactos y metas asociados.

El PND no hace alusión específica a los Planes Nacionales de la RRI, en cambio retoma sus componentes en la política de estabilización presentada por el gobierno, en donde menciona algunos de los planes nacionales bajo el denominativo de Planes Sectoriales Nacionales (PNS).

En referencia a los planes de infraestructura como el Plan Nacional de Vías para la Integración Regional (PNVIR), el PND lo acoge y procede a proponer su implementación mediante su inclusión en el Pacto por el transporte, por la construcción de paz y la descentralización, según la focalización territorial, la mejora de la red vial regional y la logística para la competitividad y la integración regional; es así que incrementa en $400 \mathrm{~km}$ la meta de vía terciaria mejorada y construida, mientras reduce los recursos previstos para este plan. En cuanto al plan de infraestructura de Riego y Drenaje, se evidencia la acogida de su base de formulación con

$7 \quad$ Las ZEll buscan una intervención integral que incluya la prestación de servicios sociales y de medidas reforzadas de protección de la población en regiones afectadas por la criminalidad que afecte la seguridad nacional 
modificaciones, según la aprobación de la política de Adecuación de Tierras, priorizando zonas de conflicto y la articulación con otras iniciativas regionales.

En lo relacionado con el plan de infraestructura eléctrica y conectividad (PNER), sus objetivos son mencionados en el Pacto por el emprendimiento y por la construcción de paz, según su rol necesario para el desarrollo del campo y la estabilización de los territorios. Sin embargo, estos no establecen metas diferenciadas para lo rural y las zonas priorizadas, además, no se enfatiza en actuar sobre zonas no interconectadas y pasa inadvertida la meta de conexión a internet de alta velocidad en las cabeceras municipales de los 170 municipios PDET, puesto que la línea de base del PND especifica que el $100 \%$ ya cuenta con dicha conexión.

Frente a los planes de desarrollo social, el PND asume en el Pacto por la equidad lo siguiente, la creación de modelos de salud con enfoque integral y diferencial para zonas rurales dispersas, así como pasar de 1 a 4 el número de entidades territoriales con este modelo y la disminución de la tasas de mortalidad materna e infantil; a pesar de lo positivo que esto representa, aún no contribuye con el objetivo de garantizar el cierre de brechas entre el campo y la ciudad, pues los indicadores de salud no hacen la diferencia entre zonas urbanas y rurales.

En el ámbito de educación se alude a la educación rural priorizando los municipios PDET, estableciendo así el logro de una tasa de cobertura bruta del $100 \%$ en educación media en dichos municipios, en tanto la meta de educación superior se centra en el impulso de programas articulados a proyectos productivos y un énfasis en programas con modalidades flexibles.

Debido a que el PND asigna la competencia en vivienda rural al Ministerio de Vivienda, Ciudad y Territorio, es necesario considerar 
su competencia sobre el Plan Nacional de construcción de Vivienda Rural, ya que el PND le ordena la definición de una nueva política, según el diagnóstico incluido en el Pacto por la Equidad frente al déficit habitacional rural.

Frente a planes de desarrollo productivo rural, el PND no trata las metas propuestas para la implementación del AF en relación con el apoyo y financiamiento de organizaciones solidarias, su fortalecimiento en capacidades productivas y administrativas y el desarrollo de estrategias para la promoción de la asociatividad a nivel municipal, pues solo incorpora la meta de proveer fortalecimiento a 1613 esquemas asociativos rurales, así como el diseño de rutas para la inclusión de población pobre y vulnerable del sector rural, sin incluir el estímulo a las compras públicas de productos de pequeños productores.

Los objetivos de Protección Social y Garantía de Derechos de trabajadores rurales son abordados desde el incremento del número de municipios con inspección móvil de trabajo en áreas rurales, la afiliación al sistema de seguridad social, las colocaciones del Servicio Público de Empleo y la formación para el trabajo, sin discriminar entre zona rural y urbana, ni territorios PDET.

Ante el Derecho a la Alimentación, el PND incluye en el Pacto por la Equidad el incremento de la producción de alimentos mediante el desarrollo de clústeres y cadenas agroindustriales, así como el establecimiento de un Sistema de Seguridad Alimentaria y Nutricional en el marco de un nuevo Plan Nacional de Seguridad Alimentaria y Nutricional; esto sumado a las acciones específicas planteadas para regiones como Chocó, la Guajira y zonas dispersas. Es entonces que, mediante la articulación entre sus pactos, el PND busca evidenciar la interdependencia inherente a la relación de proveeduría entre individuos rurales y consumidores, pues los consumidores, más allá de un pago justo por la comida, deben 
ofrecer el lugar que el campesinado merece en la sociedad como sujetos primordiales para la dinámica económica (Pachón Ariza, Bokelmann y Ramírez Miranda, 2016).

Por lo anterior, se constata que de los indicadores establecidos en el PMI son pocos los relacionados con una estrategia o indicador del PND, lo que resulta preocupante, aunado a que dentro de los que sí cuentan con un referente presentan diferencias en su unidad de medida, alcance y seguimiento.

Si bien la libertad otorgada por el PND a distintas entidades del orden nacional para el establecimiento de planes y políticas públicas sobre temas de educación y vivienda puede resultar satisfactoria en la medida en que les permite ser el formulador y ejecutor del mismo plan, esto puede resultar limitando el desarrollo rural propuesto por el AF, ya que bien puede llegar a tener una meta igual de ambiciosa o puede reducir considerablemente la prioridad asignada a tal mérito.

Finalmente, el PND integra estrategias relacionadas con los derechos humanos, como en el Pacto por la legalidad, en el que se especifica la intención de garantizar el goce efectivo, la promoción y prevención de la vulneración de los derechos humanos, cuestión que termina por evidenciar su consideración de un enfoque en derechos humanos, el cual deberá ejecutarse a través de un Plan de Acción de Derechos Humanos (PADDHH), cuya directriz se remonta a la Convención de Viena, pero cuya construcción no ha sido abordada con éxito en gobiernos anteriores. Es así que ahora la elaboración del PADDHH deberá enfrentarse a la articulación del cúmulo de normatividad en derechos humanos vigente en Colombia, si bien esta difícil tarea no es incipiente ni mucho menos exclusivamente inherente al gobierno actual, sí es prioritaria por el estadio en el cual se encuentra el Estado colombiano (Herrera Kit, 2019). 


\section{Análisis de resultados}

Considerando que el enfoque diferencial se constituye como

(...) un desarrollo específico del derecho a la igualdad, por el cual individuos o grupos humanos en situaciones similares deben ser tratados de forma igual y aquellos individuos o grupos en situaciones distintas deben ser tratados de manera distinta, en relación proporcional a dicha diferencia. (Consejería DD. HH., 2015)

Se observa cómo la RRI busca incluir tal enfoque, pero el PND no lo aborda de lleno, en cuanto descuida la distinción entre urbano y rural; razón por la que se hace prioritario visibilizar los vacíos y riesgos frente a la garantía de los derechos de población rural para con ello diseñar herramientas de solución diferenciadas.

Dicha articulación diferenciada deberá aplicar un estudio de coherencia sobre el análisis de políticas públicas, tema frente al cual autores como Cejudo y Michel (2016) sugieren que su coherencia sea integrada desde tres dimensiones.

En primer lugar, la coherencia entre el diagnóstico, objetivos y estrategias de intervención de la política, seguido de la coherencia entre las políticas según la diversidad de intervenciones que puede ocasionar que dos o más políticas se traslapen o anulen, finalizando, con la coherencia entre sistemas de políticas que puedan llegar a complementarse o no en el alcance, sentido y tipo de intervenciones que plantean. Dichas dimensiones son clave para el ejercicio de la RRI dentro del quehacer del PND, de modo que se optimicen los recursos disponibles para el desarrollo nacional y, por ende, llegar a efectuar oportuna y acertadamente la reestructuración de la realidad rural.

Tras la lectura de lo pactado y el acervo documental disponible sobre la implementación, resulta clave la prioridad dada a la ejecución efectiva del $\mathrm{AF}$, debido a que las acciones contempladas por 
la RRI no están exentas de ser sujeto de violaciones, producto de vacíos legales; de allí que declaraciones como las relacionadas con la inalienabilidad e inembargabilidad de las tierras entregadas, las líneas de referencia establecidas respecto al plazo de exención de siete años para predios asignados por adjudicación gratuita o subsidio y la condición de retorno al Fondo de Tierras de aquellos predios que permanezcan en un estado de no explotación se adecuan al propósito de garantizar el goce de derechos de la población en referencia.

Es por esto que los agentes encargados de la implementación del AF deben prestar especial atención a misivas como las enunciadas que, además de propender por la diferenciación como principio de la implementación, hacen posible identificar tanto variaciones en el curso como resultados parciales no satisfactorios, los que, a su vez, permiten tener los insumos suficientes para ajustar su curso y propender por el alcance del estado ideal.

Es posible constatar la envergadura que cobija la naturaleza de los PDET, cuya premisa soportada en la necesidad de articular profundos cambios del orden social, económico, político y cultural sobre la base del enfoque diferencial, sirve de plataforma para la protección efectiva de los derechos humanos de la población rural, especialmente en materia de educación, salud, vivienda, propiedad, alimentación, infraestructura, desarrollo económico y convivencia.

Sin embargo, cabe aclarar que la envergadura prometedora de los PDET es de la misma magnitud que los retos que su esencia envuelve, en referencia a la tarea crítica de coordinar todos los actores presentes en el territorio para la consecución de las actividades planteadas en la RRI, la organización interinstitucional y como consecuencia la capacitación y formación suficiente de la población, para propiciar una participación responsable de las comunidades y su ejercicio del mecanismo de defensa de sus derechos. 
Es así que, sobre la magna tarea que poseen los PDET y el avance observado hasta el momento, se evidencia el gran aporte hecho por la participación de las organizaciones comunitarias en territorio, ya que su labor ha fortalecido las capacidades de los individuos rurales, generando liderazgo y sentido de pertenencia. Por lo que se hace crítica la situación de amenaza a líderes rurales, en cuanto, además, de ser intolerable su persecución por razones humanitarias; también afecta la aplicación de la RRI, dado que son ellos quienes personifican el papel de ejes articuladores esenciales para la materialización de lo planteado; de allí que la Alta Comisionada de las Naciones Unidas para los Derechos Humanos exhorte al Estado colombiano a fortalecer las garantías para el ejercicio de la labor de los defensores de los derechos humanos en espacios cívicos (HCHR, 2019).

Es entonces que, es posible afirmar que el impacto más visible de la RRI sobre la protección de los derechos humanos de la Población Rural en Colombia se soporta sobre el reconocimiento individual que esta le provee a dicho colectivo y la visibilidad que les permite participar en el desarrollo consumado de su entorno. Sin embargo, es evidente la grave situación que afronta la implementación del AF, entre otros motivos, por situaciones como las reseñadas acerca de los Planes Nacionales, los cuales componen su columna vertebral y deberían ser las herramientas disponibles en la etapa incipiente de ejecución, de manera que todas las acciones ya adelantadas se encontrasen en la misma frecuencia de los Planes, diferente al escenario actual, en el que se presentan significativas discrepancias en sus recursos, metas y objetivos.

En referencia a la revisión hecha sobre el PND y su enlace con el AF, es necesario comentar que es vital la homologación entre los indicadores que rigen el curso de estos dos compilados; si bien desde un principio no se contempló homogeneidad en sus mediciones, tras el recorrido de la implementación, resulta urgente el manejo de unidades agregadas en común para la gestión de un control más certero 
y un seguimiento más efectivo de la implementación del Acuerdo. Lo anterior no permitirá desviar la atención del veedor en logros vanos y superficiales, al permitirle centrarse en los valores y acciones que realmente llegan a impactar la actividad de las comunidades rurales, facultándole a su vez para modificar el curso de las acciones ante virajes frente a los objetivos propuestos; de allí que la exactitud con la que se realice el control de la implementación marcara la pauta de la severidad en la protección y promoción de los derechos humanos de la población rural.

Gran parte del atraso y deficiencia en la implementación se debe a la precariedad de la Jurisdicción Agraria y, por ende, del soporte legal (leyes, planes y políticas) requerido para apalancar cada uno de los ejercicios de aplicación de lo pactado en el AF, situación que responde a la necesidad imperiosa de actualizar constantemente el andamiaje estatal, según las nuevas dinámicas sociales y la constante aparición o reinterpretación de derechos.

\section{Conclusiones}

Una vez finalizado el estudio sobre el primer punto del AF y su ejecución, se hace evidente el enfoque basado en derechos humanos de la RRI; en cuanto su punto de partida, cambian de las necesidades por atender en la población hacia la presencia de sujetos con capacidad de exigir prestaciones y conductas del Estado.

Por lo anterior, la salvaguarda del enfoque diferencial en cada una de las muestras materiales de la RRI es imprescindible para la creación de un legado de convivencia y paz para las futuras generaciones, en cuanto sitúa a los individuos vulnerables como actores de primera línea para la transformación progresiva de la ruralidad en Colombia. 
Finalmente, se identifica un impacto positivo y prometedor sobre los derechos humanos de la población rural a partir de la implementación de la RRI, cuestión cuya validez depende del curso que siga dicho proceso, debido a que la reducción de metas ambiciosas, la falta de coordinación y la tardanza en la aplicación de lo formulado dejan a los individuos en un limbo capaz de vulnerar sus derechos y retornarlos al estado marginal y de olvido propio de la época de conflicto.

Posterior a la revisión de la implementación de la RRI se encuentran considerables hallazgos, por lo que resulta prioritario potenciar su ejecución y certificar la incorporación de los enfoques transversales pactados con antelación -derechos humanos, género, étnico y territorial-como mecanismo para la disminución de la desigualdad.

Por esta vía se reconoce el valor de la priorización regional, en cuanto esta práctica se erige como herramienta sustancial para la reparación de la población, el tratamiento equitativo, el balance entre regiones y la no repetición del conflicto, pues es imposible dar manejo al posconflicto y establecer un escenario de paz, restando importancia a los derechos de las comunidades más vulnerables.

\section{Referencias}

Acuerdo Final. (12 de Noviembre de 2016). Obtenido de Acuerdo final para la terminación del conflicto y la construcción de una paz estable y durader. https://www.cancilleria.gov. co/sites/default/files/Fotos2016/12.11_1.2016nuevoacuerdofinal.pdf

Alta Consejería Presidencial. (18 de Julio de 2017). Colombia Renace posconflicto. Obtenido de PDET: http://especiales.presidencia.gov.co/Documents/20170718-pdet/que-sonpdet.html

Ayuso, A. y Parada Neira, A. M. (2019). CIDOB. Obtenido de Implementación del Acuerdo Final de Paz. https://www.cidob.org/es/publicaciones/documentacion/dossiers/dossier_proceso_de_paz_en_colombia/dossier_proceso_de_paz_en_colombia/implementacion_del_acuerdo_final_de_paz

Banco Mundial. (2019). Banco Mundial. Obtenido de Población rural (\% de la población total) Colombia. https://datos.bancomundial.org/indicador/SP.RUR.TOTL.ZS?locations=CO 
Boron, V., Payán, E., MacMillan, D. y Tzanopoulos, J. (2016). Achieving sustainable development in rural areas in Colombia: Future Futurescenarios for biodiversity conservation under land use change. Land Use Policy, 59, 27-37. doi:10.1016/j.landusepol

Cárdenas Pinzón, j. I. y Vallejo Zamudio, L. E. (30 de Noviembre de 2015). Agricultura y desarrollo rural en Colombia 2011-2013: una aproximación. Apuntes del CENES, 35(62), 87-123.

Cejudo, G. y Michel, C. (2016). Coherencia y políticas públicas: Metas, instrumentos y poblaciones objetivo. Gestión y política pública, 1.

CEPAL. (2013). Comisión Económica para América Latina y el Caribe. Obtenido de Definición de población urbana y rural utilizadas en los censos de los países latinoamericanos. https://www.cepal.org/sites/default/files/def_urbana_rural.pdf

CINEP - CERAC. (Junio de 2019). Centro de Investigación y Educación Popular / Programa por la Paz. Obtenido de Sexto informe de verificación de la implementación del Acuerdo Final de Paz en Colombia para los Verificadores Internacionales Felipe González y José Mujica. https://docs.google.com/viewerng/viewer?url=https://www.cinep.org. co/Home2/images/csivi/V2-Sexto_informe_trimestral_Secretara_Tcnica.pdf

Consejería DD. HH. (Diciembre de 2015). Obtenido de Estrategia nacional apra la garantía de Ios Derechos Humanos 2014-2034. http://www.derechoshumanos.gov.co/Observatorio/Publicaciones/Documents/2014/140815-estrategia_web.pdf

DAPRE. (2018). Departamento Administrativo de la Presidencia de la República. Obtenido de Informe de Rendición de Cuentas Construcción de Paz. https://dapre.presidencia. gov.co/dapre/DocumentosPlaneacion/Dapre-informe-individual-implementacion-pazNoviembre2016-May02018.pdf

DNP. (Diciembre de 2014). Misión para la transformación del Campo. Obtenido de Definición de Categorías de Ruralidad. https://colaboracion.dnp.gov.co/CDT/Estudios\%20 Econmicos/2015ago6\%20Documento\%20de\%20Ruralidad\%20-\%20DDRS-MTC.pdf

DNP. (Abril de 2017). Departamento Nacional de Planeación. Obtenido de Panorámica Regional. https://colaboracion.dnp.gov.co/CDT/Prensa/Publicaciones/ Publicaci\%C3\%B3n\%20lpm\%20deptal.pdf

Gallego Dávila, J., Azcárate, J., \& Kornov, L. (2019). Strategic Environmental Assessment for development programs and sustainability transition in the Colombian post-conflict context. Environmental Impact Assessment Review, 35-42. doi:doi.org/10.1016/j. eiar.2018.10.002

Gobierno de Colombia. (2016). Departamento Nacional de Planeación. Obtenido de Plan Marco de Implementación. https://colaboracion.dnp.gov.co/CDT/ Conpes/Econ\%C3\%B3micos/3932_Anex0\%20B_Plan\%20Marco\%20de\%20 Implementaci\%C3\%B3n\%20(PMI).pdf

HCHR. (Febrero de 2019). ONU Derechos Humanos Colombia. Obtenido de Situación de los derechos humanos en Colombia. https://www.hchr.org.co/index.php/informes-y- 
documentos/informes-anuales/9017-informe-del-alto-comisionado-de-las-nacionesunidas-para-los-derechos-humanos-sobre-la-situacion-de-derechos-humanos-encolombia-durante-el-ano-2018

Herrera Kit, P. (08 de Octubre de 2019). De la continuidad y la coherencia en la ruta de acción del gobierno Duque en materia de derechos humanos. ZERO.

Instituto Kroc. (Febrero de 2019). Kroc Institute for International Peace Studies. Obtenido de Informe 3 del Instituto Kroc. https://kroc.nd.edu/assets/315919/190408 actualizacio_n_informe_3_instituto_kroc_feb19.pdf

Locatelli, B., Aldunce, P., Fallot, A., \& Le Coq, J. F. (Octubre de 2017). Research on Climate Change Policies and Rural Development in Latin America: Scope and Gaps. Sustainability, 9, 1817. doi:10.3390/su9101831

Londoño Botero, R. (03 de Julio de 2017). Razón pública. Obtenido de Los programas de desarrollo territorial: ¿qué son y cómo van funcionar?: https://www.razonpublica.com/index.php/regiones-temas-31/10373-los-programas-de-desarrollo-territorial-qu\%C3\%A9-son-y-c\%C3\%B3mo-van-funcionar.html.

Oficina del Alto Comisionado para la Paz. (2019). Oficina del Alto Comisionado para la Paz. Obtenido de Acuerdo Política de desarrollo agrario integral. http://www.altocomisionadoparalapaz.gov.co/Documents/informes-especiales/abc-del-proceso-de-paz/ politica-de-desarrollo-agrario-integral.html

Pachón Ariza, F., Bokelmann, W. y Ramírez Miranda, C. (Agosto de 2016). Rural development thinking, moving from the green revolution to food sovereignty. Agronomía Colombiana, 32(2), 267-276. doi:10.15446/agron.colomb.v34n2.56639

PND. (2018). Departamento Nacional de Planeación. Obtenido de Plan Nacional de Desarrollo 2018 - 2022 "Pacto por Colombia, Pacto por la Equidad". https://colaboracion. dnp.gov.co/CDT/Prensa/Articulado-Plan-Nacional-de-Desarrollo-2018-2022-Pactopor-Colombia-Pacto-por-la-Equidad.pdf

Procuraduría General de la Nación. (Agosto de 2019). Obtenido de Primer Informe al Congreso sobre el estado de Avance de la Implementación del Acuerdo de Paz 2016-2019. https://www.procuraduria.gov.co/portal/media/file/Primer\%20informe\%20al\%20Congreso\%20sobre\%20el\%20estado\%20de\%20avance\%20de\%20 la\%20Implementaci\%C3\%B3n\%20del\%20Acuerdo\%20de\%20Paz\%202016\%20 $-\% 202019 . p d f$

Secretaría Técnica del Componente Internacional de Verificación CINEP/PPP-CERAC. (Junio de 2019). Cinep/Programa por la Paz. Obtenido de Informe de verificación de la implementación del Acuerdo Final de Paz en Colombia para los Verificadores Internacionales Felipe González y José Mujica. https://docs.google.com/viewerng/viewer?url=https:// www.cinep.org.co/Home2/images/csivi/V2-Sexto_informe_trimestral_Secretara_Tcnica.pdf 